\title{
Boundedness of composition operators on Morrey spaces and weak Morrey spaces
}

\author{
Naoya Hatano ${ }^{1,2,3^{*}}$, Masahiro Ikeda ${ }^{2,1}$, Isao Ishikawa ${ }^{2,4}$ and Yoshihiro Sawano ${ }^{2,3}$
}

\author{
"Correspondence: \\ n.hatano.chuo@gmail.com \\ ${ }^{1}$ Department of Mathematics, \\ Faculty of Science and Technology, \\ Keio University, 3-14-1 Hiyoshi, \\ Kohoku-ku, Yokohama 223-8522, \\ Japan \\ ${ }^{2}$ Center for Advanced Intelligence \\ Project, RIKEN, Tokyo, Japan \\ Full list of author information is \\ available at the end of the article
}

\begin{abstract}
In this study, we investigate the boundedness of composition operators acting on Morrey spaces and weak Morrey spaces. The primary aim of this study is to investigate a necessary and sufficient condition on the boundedness of the composition operator induced by a diffeomorphism on Morrey spaces. In particular, detailed information is derived from the boundedness, i.e., the bi-Lipschitz continuity of the mapping that induces the composition operator follows from the continuity of the composition mapping. The idea of the proof is to determine the Morrey norm of the characteristic functions, and employ a specific function composed of a characteristic function. As this specific function belongs to Morrey spaces but not to Lebesgue spaces, the result reveals a new phenomenon not observed in Lebesgue spaces. Subsequently, we prove the boundedness of the composition operator induced by a mapping that satisfies a suitable volume estimate on general weak-type spaces generated by normed spaces. As a corollary, a necessary and sufficient condition for the boundedness of the composition operator on weak Morrey spaces is provided.
\end{abstract}

MSC: Primary 42B35; secondary 47B33; tertiary 46E30

Keywords: Composition operators; Boundedness; Morrey spaces; Weak Morrey spaces

\section{Introduction}

In this study, we investigate the boundedness of composition operators on Morrey spaces and weak Morrey spaces. The composition operator $C_{\varphi}$ induced by a mapping $\varphi$ is a linear operator defined by $C_{\varphi} f \equiv f \circ \varphi$, where $f \circ \varphi$ represents the function composition. The composition operator is also called the Koopman operator in the fields of dynamical systems, physics, and engineering [12]. Recently, it has attracted attention in various scientific fields $[10,11]$. It becomes more and more important recently to prove the properties of composition operators mathematically.

Let $(X, \mu)$ be a $\sigma$-finite measure space, and $L^{0}(X, \mu)$ be the set of all $\mu$-measurable functions on $X$. We provide a precise definition of the composition operators induced by a measurable map $\varphi: X \rightarrow X$.

Definition 1.1 (Composition operator) Let $\varphi: X \rightarrow X$ be a measurable map, and assume that $\varphi$ is nonsingular, namely, $\mu\left(\varphi^{-1}(E)\right)=0$ for each $\mu$-measurable null set $E$. Let $V$ and

(c) The Author(s) 2021. This article is licensed under a Creative Commons Attribution 4.0 International License, which permits use, sharing, adaptation, distribution and reproduction in any medium or format, as long as you give appropriate credit to the original author(s) and the source, provide a link to the Creative Commons licence, and indicate if changes were made. The images or other third party material in this article are included in the article's Creative Commons licence, unless indicated otherwise in a credit line to the material. If material is not included in the article's Creative Commons licence and your intended use is not permitted by statutory regulation or exceeds the permitted use, you will need to obtain permission directly from the copyright holder. To view a copy of this licence, visit http://creativecommons.org/licenses/by/4.0/. 
$W$ be function spaces contained in $L^{0}(X, \mu)$. The composition operator $C_{\varphi}$ is the linear operator from $W$ to $V$ such that its domain is $\mathcal{D}\left(C_{\varphi}\right) \equiv\{h \in W: h \circ \varphi \in V\}$, and $C_{\varphi} f \equiv f \circ \varphi$ for $f \in \mathcal{D}\left(C_{\varphi}\right)$.

Subsequently, we employ the result obtained by Singh [16] for the boundedness of the composition operator on the Lebesgue space $L^{p}(X, \mu)$.

Singh [16] provided the following necessary and sufficient condition for the map $\varphi$ to generate a bounded mapping acting on Lebesgue spaces:

Theorem $1.2([16])$ Let $0<p<\infty$. Then, the composition operator $C_{\varphi}$ induced by $\varphi: X \rightarrow$ $X$ is bounded on the Lebesgue space $L^{p}(X, \mu)$ if and only if there exists a constant $K=K(\varphi)$ such that for all $\mu$-measurable sets $E$ in $\mathbb{R}^{n}$,

$$
\mu\left(\varphi^{-1}(E)\right) \leq K \mu(E)
$$

In this case, the operator norm is given by

$$
\left\|C_{\varphi}\right\|_{L^{p} \rightarrow L^{p}}=\sup _{0<\mu(E)<\infty}\left(\frac{\mu\left(\varphi^{-1}(E)\right)}{\mu(E)}\right)^{\frac{1}{p}} .
$$

The boundedness of the composition operator on $L^{\infty}(X, \mu)$ easily follows from the definition. Theorem 1.2 was extended to several important function spaces, such as Lorentz spaces [1, 6], Orlicz spaces [3, 13], mixed Lebesgue spaces [5, 7], Musielak-Orlicz spaces [14], and reproducing kernel Hilbert spaces [9]. However, there are no previous results on the boundedness of composition operators acting on Morrey spaces and weak Morrey spaces.

The first aim of this study is to investigate a necessary and sufficient condition on the boundedness of the composition operator $C_{\varphi}$ on Morrey spaces. Subsequently, we discuss the boundedness of the operator on weak Morrey spaces.

Hereafter, we consider the Euclidean space $\mathbb{R}^{n} ; \mu$ is the Lebesgue measure $\mathrm{dx}$. We denote by $|E|$ the volume of a measurable set $E \subset \mathbb{R}^{n}$. Let $\chi_{A}: \mathbb{R}^{n} \rightarrow \mathbb{R}_{\geq 0}$ be an indicator function for a subset $A \subset \mathbb{R}^{n}$, which is defined as $\chi_{A}(x)=1$ if $x \in A$ and $\chi_{A}(x)=0$, otherwise.

Now, we recall the definition of Morrey spaces on $\mathbb{R}^{n}$.

Definition 1.3 (Morrey space) Let $0<q \leq p<\infty$. The Morrey space $\mathcal{M}_{q}^{p}\left(\mathbb{R}^{n}\right)$ is the space defined by

$$
\mathcal{M}_{q}^{p}\left(\mathbb{R}^{n}\right) \equiv\left\{f \in L^{0}\left(\mathbb{R}^{n}\right):\|f\|_{\left.\mathcal{M}_{q}^{p}<\infty\right\}},\right.
$$

endowed with the quasinorm

$$
\|f\|_{\mathcal{M}_{q}^{p}} \equiv \sup _{Q \in \mathcal{Q}}|Q|^{\frac{1}{p}-\frac{1}{q}}\left\|f \chi_{Q}\right\|_{L^{q}}
$$

where $\mathcal{Q}$ denotes the family of all cubes parallel to the coordinate axes in $\mathbb{R}^{n}$.

A standard argument in functional analysis shows that $\mathcal{M}_{q}^{p}\left(\mathbb{R}^{n}\right)$ is a quasi-Banach space. From the Hölder inequality, we observe that the Lebesgue space $L^{p}\left(\mathbb{R}^{n}\right)$ is embedded into the Morrey space $\mathcal{M}_{q}^{p}\left(\mathbb{R}^{n}\right)$, where $0<q \leq p<\infty$. 
Remark 1.4 Let $0<q \leq p<\infty$. Then, we have

$$
L^{p}\left(\mathbb{R}^{n}\right)=\mathcal{M}_{p}^{p}\left(\mathbb{R}^{n}\right) \subset \mathcal{M}_{q}^{p}\left(\mathbb{R}^{n}\right) .
$$

Moreover, $L^{p}\left(\mathbb{R}^{n}\right)$ is not dense in $\mathcal{M}_{q}^{p}\left(\mathbb{R}^{n}\right)$ [15].

We now state the main results of the present paper. The following theorem provides a sufficient condition on the boundedness of the composition operator $C_{\varphi}$ on the Morrey space $\mathcal{M}_{q}^{p}\left(\mathbb{R}^{n}\right)$.

Theorem 1.5 Let $0<q \leq p<\infty$. Then, the composition operator $C_{\varphi}$ induced by $\varphi: \mathbb{R}^{n} \rightarrow$ $\mathbb{R}^{n}$ is bounded on the Morrey space $\mathcal{M}_{q}^{p}\left(\mathbb{R}^{n}\right)$, if $\varphi$ is a Lipschitz map that satisfies the volume estimate

$$
\left|\varphi^{-1}(E)\right| \leq K|E|
$$

for all measurable sets $E$ in $\mathbb{R}^{n}$ and some constant $K$ independent of $E$. In particular, we obtain

$$
\left\|C_{\varphi}\right\|_{\mathcal{M}_{q}^{p} \rightarrow \mathcal{M}_{q}^{p}} \leq(\max (1, \sqrt{n} L))^{-\frac{n}{p}+\frac{n}{q}} \sup _{E: 0<|E|<\infty}\left(\frac{\left|\varphi^{-1}(E)\right|}{|E|}\right)^{\frac{1}{q}}
$$

where $L>0$ is a Lipschitz constant of $\varphi$, and the supremum is taken over all Lebesgue measurable sets $E$ satisfying $0<|E|<\infty$.

Conversely, as stated in the following theorem, if $\varphi: \mathbb{R}^{n} \rightarrow \mathbb{R}^{n}$ is a diffeomorphism, then the $\mathcal{M}_{q}^{p}\left(\mathbb{R}^{n}\right)$-boundedness of the composition operators $C_{\varphi}$ and $C_{\varphi^{-1}}$ indicates that $\varphi$ is bi-Lipschitz. Note that any bi-Lipschitz mapping satisfies the assumption of Theorem 1.5.

Theorem 1.6 Let $n \in \mathbb{N}$, and $\varphi: \mathbb{R}^{n} \rightarrow \mathbb{R}^{n}$ be a diffeomorphism in the sense that $\varphi$ and its inverse $\varphi^{-1}$ are differentiable. Suppose $0<q<p<\infty$, or $q=p$ and $n=1$. If the composition operators $C_{\varphi}$ and $C_{\varphi^{-1}}$ induced by maps $\varphi$ and $\varphi^{-1}$, respectively, are bounded on $\mathcal{M}_{q}^{p}\left(\mathbb{R}^{n}\right)$, then $\varphi$ is bi-Lipschitz.

Remark 1.7 In the case where $p=q, n=1$ and $\varphi$ is a diffeomorphism, Theorem 1.6 is equivalent to Theorem 1.2. Unless $n=1$, condition $q<p$ is essential in the following sense. If $n \geq 2$ and $q=p$, then the same conclusion as in Theorem 1.6 fails. We present a counterexample in Example 3.3 in Sect. 4. Noting that the Morrey space $\mathcal{M}_{p}^{p}\left(\mathbb{R}^{n}\right)$ coincides with the Lebesgue space $L^{p}\left(\mathbb{R}^{n}\right)$ (see Remark 1.4), we observe a new phenomenon from Theorem 1.6. Whenever the composition operator generated by a diffeomorphism $\varphi$ is an isomorphism in the Morrey space $\mathcal{M}_{q}^{p}\left(\mathbb{R}^{n}\right)$ with $0<q<p<\infty, \varphi$ must be bi-Lipschitz continuous. This phenomenon cannot be observed as is seen from Example 3.3. Unlike other function spaces, Morrey spaces are not rearrangement invariant. This is because some geometric conditions are included in the definition of the norm. Due to these geometric conditions, it seems natural for the Lipschitz continuity to come into play.

Subsequently, we investigate the characterization of the boundedness of the composition operators acting on weak Morrey spaces, which are defined as follows: 
Definition 1.8 (Weak Morrey space) Let $0<q \leq p<\infty$. The weak Morrey space $\mathrm{W} \mathcal{M}_{q}^{p}\left(\mathbb{R}^{n}\right)$ is the space defined by

$$
\mathrm{WM}_{q}^{p}\left(\mathbb{R}^{n}\right) \equiv\left\{f \in L^{0}\left(\mathbb{R}^{n}\right):\|f\|_{\mathrm{WM}_{q}^{p}}<\infty\right\}
$$

endowed with the quasinorm

$$
\|f\|_{\mathrm{W} \mathcal{M}_{q}^{p}} \equiv \sup _{\lambda>0} \lambda\left\|\chi_{\left\{x \in \mathbb{R}^{n}:|f(x)|>\lambda\right\}}\right\|_{\mathcal{M}_{q}^{p}}
$$

Another standard argument in functional analysis shows that $\mathcal{M}_{q}^{p}\left(\mathbb{R}^{n}\right)$ is a quasi-Banach space. The weak Morrey space $\mathrm{W} \mathcal{M}_{q}^{p}\left(\mathbb{R}^{n}\right)$ has the following basic properties:

Remark 1.9 Let $0<q<p<\infty$. Then, we have

$$
\mathcal{M}_{q}^{p}\left(\mathbb{R}^{n}\right) \subset \mathrm{WM}_{q}^{p}\left(\mathbb{R}^{n}\right), \quad \mathrm{W}_{\mathcal{M}}^{p}\left(\mathbb{R}^{n}\right)=\mathrm{W} L^{p}\left(\mathbb{R}^{n}\right) .
$$

The following theorem provides a necessary and sufficient condition on the boundedness of the composition operator on weak Morrey spaces.

Theorem 1.10 Let $0<q \leq p<\infty$, and let $\varphi: \mathbb{R}^{n} \rightarrow \mathbb{R}^{n}$ be a measurable function. Then, $\varphi$ generates the composition operator $C_{\varphi}$ which is bounded on the weak Morrey space $\mathrm{WM}_{q}^{p}\left(\mathbb{R}^{n}\right)$ if and only if there exists a constant $K$ such that for all measurable sets $E$ in $\mathbb{R}^{n}$, the estimate

$$
\left\|\chi_{\varphi^{-1}(E)}\right\|_{\mathcal{M}_{q}^{p}} \leq K\left\|\chi_{E}\right\|_{\mathcal{M}_{q}^{p}}
$$

holds. In particular, we obtain

$$
\left\|C_{\varphi}\right\|_{\mathrm{W} \mathcal{M}_{q}^{p} \rightarrow \mathrm{W} \mathcal{M}_{q}^{p}}=\sup _{E} \frac{\left\|\chi_{\varphi^{-1}(E)}\right\|_{\mathcal{M}_{q}^{p}}}{\left\|\chi_{E}\right\|_{\mathcal{M}_{q}^{p}}},
$$

where the supremum is taken over all measurable sets $E$ in $\mathbb{R}^{n}$ with $0<\left\|\chi_{E}\right\|_{\mathcal{M}_{q}^{p}}<\infty$.

\section{Remark 1.11}

(1) Theorem 1.10 indicates that the composition operator $C_{\varphi}$ is bounded on weak Morrey spaces once it is bounded on Morrey spaces (see Sect. 4 for more).

(2) The conclusion of the case $q=p$ in this theorem was provided in [2].

(3) Theorem 1.10 is a special case of Theorem 1.13 below.

In fact, we will establish the boundedness of the composition operator in a more general framework.

Definition 1.12 Let $\left(B\left(\mathbb{R}^{n}\right),\|\cdot\|_{B}\right)$ be a linear subspace of $L^{0}\left(\mathbb{R}^{n}\right)$ such that $\|f \mid\|_{B}=\|f\|_{B}$ for all $f \in B\left(\mathbb{R}^{n}\right)$. The weak-type space $\left(\mathrm{W} B\left(\mathbb{R}^{n}\right),\|\cdot\|_{\mathrm{W} B}\right)$ of $B$ is defined by

$$
\mathrm{W} B\left(\mathbb{R}^{n}\right) \equiv\left\{f \in L^{0}\left(\mathbb{R}^{n}\right):\|f\|_{\mathrm{W} B}<\infty\right\},
$$


endowed with the quasinorm

$$
\left.\|f\|_{\mathrm{W} B} \equiv \sup _{\lambda>0} \lambda \| \chi_{\{x \in \mathbb{R}}:|f(x)|>\lambda\right\} \|_{B}
$$

For example, the space $\mathrm{W} L^{p}\left(\mathbb{R}^{n}\right)$ is the weak Lebesgue space whose norm is given by $\|f\|_{L_{L}}=\sup _{\lambda>0} \lambda\left\|\chi_{f^{-1}((\lambda, \infty])}\right\|_{L^{p}}$ (see [8, Chap. 1] for more).

Now, we can rewrite Theorem 1.10 as follows:

Theorem 1.13 Let $\left(B\left(\mathbb{R}^{n}\right),\|\cdot\|_{B}\right)$ be a normed space. Then, the composition induced by $\varphi$ is bounded on the weak-type space $\left(\mathrm{W} B\left(\mathbb{R}^{n}\right),\|\cdot\|_{\mathrm{W} B}\right)$ if and only if there exists a constant $K$ such that for all measurable sets $E$ in $\mathbb{R}^{n}$, the estimate

$$
\left\|\chi_{\varphi^{-1}(E)}\right\|_{B} \leq K\left\|\chi_{E}\right\|_{B}
$$

holds. In particular, we obtain

$$
\left\|C_{\varphi}\right\|_{\mathrm{W} B \rightarrow \mathrm{W} B}=\sup _{E} \frac{\left\|\chi_{\varphi^{-1}(E)}\right\|_{B}}{\left\|\chi_{E}\right\|_{B}},
$$

where the supremum is taken over all measurable sets $E$ in $\mathbb{R}^{n}$ with $0<\left\|\chi_{E}\right\|_{B}<\infty$.

Here is a list of standard notation used in this paper in addition to that which has already appeared above:

- The space $L_{\mathrm{c}}^{\infty}\left(\mathbb{R}^{n}\right)$ stands for the set of all $L^{\infty}\left(\mathbb{R}^{n}\right)$ functions with compact support.

- The linear space $\mathrm{M}_{n}\left(\mathbb{R}^{n}\right)$ is the set of all $n \times n$-matrices.

- For $\alpha_{1}, \alpha_{2}, \ldots, \alpha_{n}$, the matrix $\operatorname{diag}\left(\alpha_{1}, \ldots, \alpha_{n}\right)$ is the diagonal matrix with entries $\alpha_{1}, \alpha_{2}, \ldots, \alpha_{n}$.

- The matrix $E \in \mathrm{M}_{n}\left(\mathbb{R}^{n}\right)$ denotes the identity matrix.

- For $A \in \mathrm{M}_{n}\left(\mathbb{R}^{n}\right)$, the quantity $\|A\|_{\mathrm{F}}$ denotes the Frobenius norm defined by $\sqrt{\operatorname{tr}\left(A^{\mathrm{T}} A\right)}$.

- The space $C_{\mathrm{c}}^{\infty}\left(\mathbb{R}^{n}\right)$ is the set of all smooth functions with compact support.

- For a cube $Q, \ell(Q)$ stands for its side-length.

- Let $A, B \geq 0$. Then $A \lesssim B$ and $B \gtrsim A$ mean that there exists a constant $C>0$ such that $A \leq C B$, where $C$ depends only on the parameters of importance. The symbol $A \sim B$ means that $A \lesssim B$ and $B \lesssim A$ happen simultaneously. For example, for functions $A(x)$ and $B(x)$ of $x$, we use shorthand $A(x) \lesssim B(x)$ to denote an estimate $A(x) \leq C B(x)$ with some constant $C>0$ independent of $x$. As a result, notation $A(x) \sim B(x)$ represents $A(x) \lesssim B(x)$ and $B(x) \lesssim A(x)$.

- For a differentiable vector-valued function $\varphi=\left(\varphi_{1}, \ldots, \varphi_{n}\right)^{\mathrm{T}}$ on $\mathbb{R}^{n}$, we denote by $D \varphi$ the Jacobi matrix of $\varphi$, that is,

$$
D \varphi \equiv\left(\frac{\partial \varphi_{i}}{\partial x_{j}}\right)_{1 \leq i, j \leq n} \equiv\left(\varphi_{i, j}\right)_{1 \leq i, j \leq n}
$$

The remainder of this paper is organized as follows: In Sect. 2, we prove Theorems 1.5 and 1.6. In Sect. 3, we present some examples and counterexamples of the mapping that induces the composition operator to be bounded on Morrey spaces. In Sect. 4, we prove Theorem 1.13. 


\section{Proofs of Theorems 1.5 and 1.6}

In this section, we prove Theorems 1.5 and 1.6. The proof of Theorem 1.5 is provided in Sect. 2.1. However, Theorem 1.6 is more difficult to prove. In Sect. 2.2, we reduce matters to the linear setting. We divide its proof into two steps: we consider case $p \leq n q$ in Sect. 2.3 and case $n q \leq p$ in Sect. 2.4.

\subsection{Proof of Theorem 1.5}

Proof of Theorem 1.5 A cube $Q \in \mathcal{Q}$ is fixed. We note that, according to the Lipschitz continuity of $\varphi$, the estimates

$$
\operatorname{diam}(\varphi(Q)):=\sup _{x, \tilde{x} \in Q}|\varphi(x)-\varphi(\tilde{x})| \leq L \sup _{x, \tilde{x} \in Q}|x-\tilde{x}|=\sqrt{n} L \ell(Q)
$$

hold; thus, there exist cubes $Q_{1}, Q_{2} \in \mathcal{Q}$ such that

$$
Q_{1} \supset Q, \quad Q_{2} \supset \varphi(Q), \quad\left|Q_{1}\right|=\left|Q_{2}\right|=(\max (1, \sqrt{n} L))^{n}|Q| .
$$

Since $\varphi$ satisfies condition (1.2), we can apply the $L^{q}\left(\mathbb{R}^{n}\right)$-boundedness of the composition operators (Theorem 1.2) to obtain

$$
\begin{aligned}
& |Q|^{\frac{1}{p}-\frac{1}{q}}\left(\int_{Q}|f(\varphi(x))|^{q} \mathrm{~d} x\right)^{\frac{1}{q}} \\
& \quad \leq|Q|^{\frac{1}{p}-\frac{1}{q}}\left(\int_{\mathbb{R}^{n}}|f(\varphi(x))|^{q} \chi_{\varphi(Q)}(\varphi(x)) \mathrm{d} x\right)^{\frac{1}{q}} \\
& \quad \leq|Q|^{\frac{1}{p}-\frac{1}{q}} \cdot\left\|C_{\varphi}\right\|_{L^{q} \rightarrow L^{q}}\left(\int_{\mathbb{R}^{n}}|f(x)|^{q} \chi_{\varphi(Q)}(x) \mathrm{d} x\right)^{\frac{1}{q}} \\
& \quad \leq\left((\max (1, \sqrt{n} L))^{-n}\left|Q_{1}\right|\right)^{\frac{1}{p}-\frac{1}{q}} \cdot\left\|C_{\varphi}\right\|_{L^{q} \rightarrow L^{q}}\left(\int_{\mathbb{R}^{n}}|f(x)|^{q} \chi_{Q_{2}}(x) \mathrm{d} x\right)^{\frac{1}{q}} \\
& \quad \leq(\max (1, \sqrt{n} L))^{-\frac{n}{p}+\frac{n}{q}}\left\|C_{\varphi}\right\|_{L^{q} \rightarrow L^{q}}\|f\|_{\mathcal{M}_{q}^{p},}
\end{aligned}
$$

which indicates that the composition operator $C_{\varphi}$ is bounded on $\mathcal{M}_{q}^{p}\left(\mathbb{R}^{n}\right)$. Moreover, by applying equation (1.1), we obtain (1.3), which completes the proof of the theorem.

\subsection{Reduction of the diffeomorphism to the linear setting}

In this subsection, by applying the following lemma (Lemma 2.1), we reduce the diffeomorphism $\varphi: \mathbb{R}^{n} \rightarrow \mathbb{R}^{n}$ in Theorem 1.6 to the linear mapping $D \varphi: \mathbb{R}^{n} \rightarrow \mathrm{M}_{n}(\mathbb{R})$. By the estimate of the singular values of the Jacobi matrix $D \varphi$, we will show that $\varphi$ is bi-Lipschitz (see Proposition 2.6 below).

Lemma 2.1 Let $0<q \leq p<\infty$. Suppose that a diffeomorphism $\varphi: \mathbb{R}^{n} \rightarrow \mathbb{R}^{n}$ induces a bounded composition operator $C_{\varphi}$ from $\mathcal{M}_{q}^{p}\left(\mathbb{R}^{n}\right)$ to itself. Then, there exists a positive constant $K>0$ such that

$$
\left\|C_{D \varphi\left(x_{0}\right)} f\right\|_{\mathcal{M}_{q}^{p}}=\left\|f\left(D \varphi\left(x_{0}\right) \cdot\right)\right\|_{\mathcal{M}_{q}^{p}} \leq K\|f\|_{\mathcal{M}_{q}^{p}}
$$


for all $x_{0} \in \mathbb{R}^{n}$ and $f \in \mathcal{M}_{q}^{p}\left(\mathbb{R}^{n}\right)$. In particular, the operator norm of $\left\|C_{D \varphi\left(x_{0}\right)}\right\|$ is bounded above by a constant independent of $x_{0}$.

Proof of Lemma 2.1 Set $K \equiv\left\|C_{\varphi}\right\|_{\mathcal{M}_{q}^{p} \rightarrow \mathcal{M}_{q}^{p}}<\infty$. First, we prove the assertion for $f \in$ $C_{\mathrm{c}}^{\infty}\left(\mathbb{R}^{n}\right)$. Let $t>0$. We calculate

$$
\begin{aligned}
\left\|f\left(\frac{\varphi\left(x_{0}+t \cdot\right)-\varphi\left(x_{0}\right)}{t}\right)\right\|_{\mathcal{M}_{q}^{p}} & =t^{-\frac{n}{p}}\left\|f\left(\frac{\varphi(\cdot)-\varphi\left(x_{0}\right)}{t}\right)\right\|_{\mathcal{M}_{q}^{p}} \\
& \leq K t^{-\frac{n}{p}}\left\|f\left(\frac{\cdot-\varphi\left(x_{0}\right)}{t}\right)\right\|_{\mathcal{M}_{q}^{p}} \\
& =K\|f\|_{\mathcal{M}_{q}^{p} .}
\end{aligned}
$$

By letting $t \rightarrow 0$, we obtain the desired result for $f \in C_{\mathrm{c}}^{\infty}\left(\mathbb{R}^{n}\right)$.

Let $f \in L_{\mathrm{c}}^{\infty}\left(\mathbb{R}^{n}\right)$. Then, for any $p \in(0, \infty)$, we can choose a sequence $\left\{f_{j}\right\}_{j=1}^{\infty}$ of compactly supported smooth functions such that $f_{j}$ converges to $f$ in $L^{p}\left(\mathbb{R}^{n}\right)$ as $j \rightarrow \infty$. By passing to a subsequence, we may assume that $f_{j}$ converges to $f$, almost everywhere in $\mathbb{R}^{n}$ as $j \rightarrow \infty$. Thus, by the Fatou lemma, the inequality

$$
\left\|f\left(D \varphi\left(x_{0}\right) \cdot\right)\right\|_{\mathcal{M}_{q}^{p}} \leq \liminf _{j \rightarrow \infty}\left\|f_{j}\left(D \varphi\left(x_{0}\right) \cdot\right)\right\|_{\mathcal{M}_{q}^{p}}
$$

holds. As we have proved the assertion for $f_{j}$, we have

$$
\left\|f_{j}\left(D \varphi\left(x_{0}\right) \cdot\right)\right\|_{\mathcal{M}_{q}^{p}} \leq K\left\|f_{j}\right\|_{\mathcal{M}_{q}^{p}}
$$

Since $L^{p}\left(\mathbb{R}^{n}\right)$ is embedded into $\mathcal{M}_{q}^{p}\left(\mathbb{R}^{n}\right)$ (see Remark 1.4), $f_{j}$ converges to $f$ in $\mathcal{M}_{q}^{p}\left(\mathbb{R}^{n}\right)$ as $j \rightarrow \infty$. Consequently,

$$
\liminf _{j \rightarrow \infty}\left\|f_{j}\right\|_{\mathcal{M}_{q}^{p}}=\|f\|_{\mathcal{M}_{q}^{p}}
$$

By combining these observations, the following estimate holds:

$$
\left\|f\left(D \varphi\left(x_{0}\right) \cdot\right)\right\|_{\mathcal{M}_{q}^{p}} \leq K\|f\|_{\mathcal{M}_{q}^{p}}
$$

Finally, let $f \in \mathcal{M}_{q}^{p}\left(\mathbb{R}^{n}\right)$. For $k \in \mathbb{N}$, we define an element $f_{k} \in L_{c}^{\infty}\left(\mathbb{R}^{n}\right)$ by

$$
f_{k}(x) \equiv f(x) \chi_{[-k, k]^{n}}(x) \chi_{[0, k]}(|f(x)|) \quad\left(x \in \mathbb{R}^{n}\right) .
$$

Then, we have

$$
\left\|f_{k}\left(D \varphi\left(x_{0}\right) \cdot\right)\right\|_{\mathcal{M}_{q}^{p}} \leq K\left\|f_{k}\right\|_{\mathcal{M}_{q}^{p}} \leq K\|f\|_{\mathcal{M}_{q}^{p}}
$$

according to the previous paragraph. By using the Fatou lemma again, we obtain

$$
\left\|f\left(D \varphi\left(x_{0}\right) \cdot\right)\right\|_{\mathcal{M}_{q}^{p}} \leq K\|f\|_{\mathcal{M}_{q}^{p}}
$$

as required. 
Let $\varphi: \mathbb{R}^{n} \rightarrow \mathbb{R}^{n}$ be a diffeomorphism and $D \varphi: \mathbb{R}^{n} \rightarrow \mathrm{M}_{n}(\mathbb{R})$ be its Jacobi matrix. For $x_{0} \in \mathbb{R}^{n}$, the Jacobi matrix $D \varphi\left(x_{0}\right)$ can be decomposed by the singular value decomposition (see Lemma 2.2 below) as

$$
D \varphi\left(x_{0}\right)=U \Sigma V
$$

where $\Sigma=\Sigma\left(x_{0}\right)=\operatorname{diag}\left(\alpha_{1}\left(x_{0}\right), \ldots, \alpha_{n}\left(x_{0}\right)\right)$ is a diagonal matrix with having positive components satisfying $\alpha_{1}\left(x_{0}\right) \leq \cdots \leq \alpha_{n}\left(x_{0}\right)$, and $U=U\left(x_{0}\right)$ and $V=V\left(x_{0}\right)$ are orthogonal matrices.

Lemma 2.2 (Singular value decomposition) Let $A$ be an $n \times n$ real regular matrix, and $\alpha_{1}, \ldots, \alpha_{n}>0$ be the singular values of $A$. Then, there exist orthogonal matrices $U$ and $V$ such that

$$
U A V=\operatorname{diag}\left(\alpha_{1}, \ldots, \alpha_{n}\right) .
$$

Now, by the definition of the Morrey norm $\|\cdot\|_{\mathcal{M}_{q}^{p}}$, and a simple computation, we have the equivalence

$$
n^{\frac{n}{p}-\frac{n}{q}}\left\|C_{\Sigma\left(x_{0}\right)}\right\|_{\mathcal{M}_{q}^{p} \rightarrow \mathcal{M}_{q}^{p}} \leq\left\|C_{D \varphi\left(x_{0}\right)}\right\|_{\mathcal{M}_{q}^{p} \rightarrow \mathcal{M}_{q}^{p}} \leq n^{-\frac{n}{p}+\frac{n}{q}}\left\|C_{\Sigma\left(x_{0}\right)}\right\|_{\mathcal{M}_{q}^{p} \rightarrow \mathcal{M}_{q}^{p}}
$$

Here, the operator norms $\|\cdot\|_{\mathcal{M}_{q}^{p} \rightarrow \mathcal{M}_{q}^{p}}$ of the composition operators induced by the orthogonal matrices are bounded above by a constant independent of the selection of the rotation matrices. Therefore, we have the following lemma:

Lemma 2.3 Let $0<q \leq p<\infty$. Suppose that a diffeomorphism $\varphi: \mathbb{R}^{n} \rightarrow \mathbb{R}^{n}$, induces a bounded composition operator $C_{\varphi}$ on $\mathcal{M}_{q}^{p}\left(\mathbb{R}^{n}\right)$. Let $\alpha_{1}\left(x_{0}\right), \ldots, \alpha_{n}\left(x_{0}\right)$ be the singular values of $D \varphi\left(x_{0}\right)$, and let us denote $\Sigma\left(x_{0}\right):=\operatorname{diag}\left(\alpha_{1}\left(x_{0}\right), \ldots, \alpha_{n}\left(x_{0}\right)\right)$. Then, the operator norm of $C_{\Sigma\left(x_{0}\right)}$ is bounded above by a constant independent of $x_{0}$.

Proposition 2.4 Let $0<q \leq p<\infty, x_{0} \in \mathbb{R}^{n}$, and $\varphi: \mathbb{R}^{n} \rightarrow \mathbb{R}^{n}$ be diffeomorphism. If the composition operators $C_{\varphi}$ and $C_{\varphi^{-1}}$ induced by $\varphi$ and $\varphi^{-1}$, respectively, are bounded on the Morrey space $\mathcal{M}_{q}^{p}\left(\mathbb{R}^{n}\right)$, then we have

$$
\prod_{k=1}^{n} \alpha_{k}\left(x_{0}\right) \sim 1,
$$

where $\alpha_{1}\left(x_{0}\right), \ldots, \alpha_{n}\left(x_{0}\right)$ are the singular values of $D \varphi\left(x_{0}\right)$.

This proposition can be proved by combining Lemmas 2.1 and 2.5 below.

Lemma 2.5 Let $0<q \leq p<\infty$ and $\left\{a_{1}, \ldots, a_{n}\right\} \subset \mathbb{R}_{>0}$ be a positive sequence and set $D \equiv$ $\operatorname{diag}\left(a_{1}, \ldots, a_{n}\right)$. Then, the following estimate holds:

$$
\prod_{k=1}^{n} a_{k} \geq\left\|C_{D}\right\|_{\mathcal{M}_{q}^{p} \rightarrow \mathcal{M}_{q}^{p}}^{-p}
$$


Proof We introduce matrix $W \in \mathrm{M}_{n}(\mathbb{R})$ corresponding to the transform

$$
\left(x_{1}, x_{2}, \ldots, x_{n}\right) \mapsto\left(x_{2}, x_{3}, \ldots, x_{n}, x_{1}\right) .
$$

Then, for any $k \in\{1, \ldots, n\}$, we observe that

$$
W^{-k} D W^{k}=\operatorname{diag}\left(a_{n-k+1}, a_{n-k+2}, \ldots, a_{n}, a_{1}, a_{2}, \ldots, a_{n-k}\right)
$$

holds, since $W^{k}$ maps the $l$ th elementary vector $e_{l}$ to $e_{l-k}$ if $l>k$ and $e_{l-k+n}$ otherwise.

Since $W$ leaves $\mathcal{Q}$ invariant and $D C_{W}=E$,

$$
\left\|C_{W-k D W k}\right\|_{\mathcal{M}_{q}^{p} \rightarrow \mathcal{M}_{q}^{p}}=\left\|C_{D}\right\|_{\mathcal{M}_{q}^{p} \rightarrow \mathcal{M}_{q}^{p}}
$$

hold. Noting that the identity

$$
\prod_{k=1}^{n} W^{-k} D W^{k}=\left(\prod_{k=1}^{n} a_{k}\right) E
$$

holds, we learn that the equality

$$
\left\|C_{\prod_{k=1}^{n} W^{-k} D W^{k}}\right\|_{\mathcal{M}_{q}^{p} \rightarrow \mathcal{M}_{q}^{p}}=\left(\prod_{k=1}^{n} a_{k}\right)^{-\frac{n}{p}}
$$

holds. By combining this and identity $C_{\prod_{k=1}^{n} W^{-k} D_{W^{k}}}=\prod_{k=1}^{n} C_{W^{-k} D W^{k}}$, the conclusion of this lemma is proved.

To obtain the bi-Lipschitz continuity of $\varphi$, we use the following proposition, which is obtained using the mean value theorem.

Proposition 2.6 Let $\varphi: \mathbb{R}^{n} \rightarrow \mathbb{R}^{n}$ be a diffeomorphism. Let $\alpha_{1}\left(x_{0}\right)$ be a minimal singular value of $D \varphi\left(x_{0}\right)$. If there exists a positive constant $C>0$ independent of $x_{0}$ such that for all $x_{0} \in \mathbb{R}^{n}$,

$$
\alpha_{1}\left(x_{0}\right) \geq C
$$

then the inverse function $\varphi^{-1}$ of $\varphi$ is Lipschitz.

Proof Assume $x, \tilde{x} \in \mathbb{R}^{n}$ are fixed. Since the mapping $\varphi^{-1}$ is differentiable on the line segment between $x$ and $\tilde{x}$, by the mean value theorem, we can consider point $x_{0}$ on the line segment between $x$ and $\tilde{x}$ and obtain

$$
\left|\varphi^{-1}(x)-\varphi^{-1}(\tilde{x})\right|=\left\|D \varphi^{-1}\left(x_{0}\right)\right\|_{F}|x-\tilde{x}| .
$$

Now, using the decomposition (2.1), we can calculate

$$
\left\|D \varphi^{-1}\left(x_{0}\right)\right\|_{\mathrm{F}}=\left(\sum_{j=1}^{n}\left|\frac{1}{\alpha_{j}\left(x_{0}\right)}\right|^{2}\right)^{\frac{1}{2}} \leq \frac{\sqrt{n}}{C} .
$$

Consequently, we obtain the Lipschitz continuity of $\varphi^{-1}$. 
According to this proposition, to obtain Theorem 1.6, it suffices to show that there exists a positive constant $C>0$ such that for each $x_{0} \in \mathbb{R}^{n}$, estimate (2.2) holds. We divide the proof of (2.2) into the two cases $p \leq n q$ and $n q \leq p$.

\subsection{Proof of (2.2) in the case $p \leq n q$}

To obtain estimate (2.2), we introduce a parameter $m$, we estimate the operator norm of the diagonal matrices $\Sigma\left(x_{0}\right)$ in the decomposition (2.1) as follows using Lemma 2.7 and Proposition 2.8 below.

Lemma 2.7 Let $n \in \mathbb{N}$ and $0<q \leq p<\infty$ satisfy $p \leq n q$. Assume in addition that the sequence $\left\{a_{j}\right\}_{j=0}^{n-1}$ satisfies $a_{0}=1 \leq a_{1} \leq \cdots \leq a_{n-1}$. Then,

$$
\left\|\chi_{[0,1] \times \prod_{j=1}^{n-1}\left[0, a_{j}\right]}\right\|_{\mathcal{M}_{q}^{p}}=\left(\prod_{j=1}^{m-1} a_{j}^{\frac{1}{q}}\right) a_{m-1}^{\frac{n}{p}-\frac{m}{q}}
$$

where $m \in[2, n]$ is the unique integer satisfying $0<q \leq \frac{n}{m} q \leq p \leq \frac{n}{m-1} q<\infty$.

Proof Since we have to consider only cubes of form $[0, R]^{n}$ for $R>0$ as the candidates for supremum in the Morrey norm $\|\cdot\|_{\mathcal{M}_{q}^{p}}$, we have identity

$$
\left\|\chi_{[0,1] \times \prod_{j=1}^{n-1}\left[0, a_{j}\right]}\right\|_{\mathcal{M}_{q}^{p}}=\sup _{R>0} R^{\frac{n}{p}-\frac{n}{q}} \prod_{j=0}^{n-1} \min \left(a_{j}, R\right)^{\frac{1}{q}}
$$

By considering the case of $R=1, a_{1}, \ldots, a_{n-1}$, we can determine the supremum on the righthand side of the above identity as follows:

$$
\left\|\chi_{[0,1] \times \prod_{j=1}^{n-1}\left[0, a_{j}\right]}\right\|_{\mathcal{M}_{q}^{p}}=\max \left\{\left(\prod_{j=0}^{k-1} a_{j}\right) a_{k}^{\frac{n}{p}-\frac{k}{q}}: k=0,1, \ldots, n-1\right\}
$$

Here, according to assumption $p \leq \frac{n}{m-1} q$, we observe that

$$
a_{1}^{\frac{1}{q}} a_{1}^{\frac{n}{p}-\frac{2}{q}}=a_{1}^{\frac{n}{p}-\frac{1}{q}} \geq 1
$$

when $m=2$, and

$$
\left(\prod_{j=1}^{m-1} a_{j}\right)^{\frac{1}{q}} a_{m-1}^{\frac{n}{p}-\frac{m}{q}} \geq\left(\prod_{j=1}^{m-2} a_{j}\right)^{\frac{1}{q}} a_{m-2}^{\frac{n}{p}-\frac{m-1}{q}} \geq \cdots \geq a_{1}^{\frac{n}{p}-\frac{1}{q}} \geq 1
$$

when $m>2$. Meanwhile, using the assumption $\frac{n}{m} q \leq p$, we calculate

$$
\left(\prod_{j=1}^{m-1} a_{j}\right)^{\frac{1}{q}} a_{m-1}^{\frac{n}{p}-\frac{m}{q}} \geq\left(\prod_{j=1}^{m} a_{j}\right)^{\frac{1}{q}} a_{m}^{\frac{n}{p}-\frac{m+1}{q}} \geq \cdots \geq\left(\prod_{j=1}^{n-1} a_{j}\right)^{\frac{1}{q}} a_{n-1}^{\frac{n}{p}-\frac{n}{q}},
$$

to conclude that quantity

$$
\left(\prod_{j=1}^{m-1} a_{j}\right)^{\frac{1}{q}} a_{m-1}^{\frac{n}{p}-\frac{m}{q}}
$$


is the largest when taking the maximum in equation (2.3). Hence, this is the desired result.

Proposition 2.8 Let $n \geq m \geq 2,0<q \leq \frac{n}{m} q \leq p \leq \frac{n}{m-1} q<\infty$, and $1 \leq a_{1} \leq \cdots \leq a_{n-1}$. Then, we have

$$
\left\|C_{\operatorname{diag}\left(1, a_{1}, \ldots, a_{n-1}\right)}\right\|_{\mathcal{M}_{q}^{p} \rightarrow \mathcal{M}_{q}^{p}} \geq\left(\prod_{j=1}^{m-1} a_{j}\right)^{-\frac{1}{q}} a_{m-1}^{-\frac{n}{p}+\frac{m}{q}} .
$$

Proof Simply using Lemma 2.7, we have

$$
\left\|\chi_{[0,1] \times \prod_{j=1}^{n-1}\left[0, R_{j}\right]}\right\|_{\mathcal{M}_{q}^{p}}=\left(\prod_{j=1}^{m-1} R_{j}\right)^{\frac{1}{q}} R_{m-1}^{\frac{n}{p}-\frac{m}{q}}
$$

and

$$
\left\|\chi_{[0,1] \times \prod_{j=1}^{n-1}\left[0, a_{j}^{-1} R_{j}\right]}\right\|_{\mathcal{M}_{q}^{p}}=\left(\prod_{j=1}^{m-1} a_{j}^{-1} R_{j}\right)^{\frac{1}{q}}\left(a_{m-1}^{-1} R_{m-1}\right)^{\frac{n}{p}-\frac{m}{q}}
$$

for $1 \leq R_{1} \leq \cdots \leq R_{n-1}$ with $1 \leq a_{1}^{-1} R_{1} \leq \cdots \leq a_{n-1}^{-1} R_{n-1}$.

Now, we prove estimate (2.2) under the assumption $p \leq n q$. It suffices to consider case $\frac{n}{m} q<p \leq \frac{n}{m-1} q$, for each $m=2, \ldots, n$ since $p \leq n q$. According to Lemma 2.3 and Proposition 2.8 , we calculate

$$
\begin{aligned}
1 & \gtrsim\left\|C_{\Sigma\left(x_{0}\right)}\right\|_{\mathcal{M}_{q}^{p} \rightarrow \mathcal{M}_{q}^{p}} \\
& \geq \alpha_{1}\left(x_{0}\right)^{-\frac{n}{p}} \prod_{i \in I}\left(\frac{\alpha_{i}\left(x_{0}\right)}{\alpha_{1}\left(x_{0}\right)}\right)^{-\frac{1}{q}} \cdot\left(\frac{\alpha_{j}\left(x_{0}\right)}{\alpha_{1}\left(x_{0}\right)}\right)^{-\frac{n}{p}+\frac{m}{q}} \\
& =\left(\alpha_{1}\left(x_{0}\right) \prod_{i \in I} \alpha_{i}\left(x_{0}\right)\right)^{-\frac{1}{q}} \alpha_{j}\left(x_{0}\right)^{-\frac{n}{p}+\frac{m}{q}},
\end{aligned}
$$

where $I$ is a subset of $\{2, \ldots, n\}$ such that $\# I=m-1$ and $j \in I$. Combining estimate (2.4) and Proposition 2.4, we have

$$
\begin{aligned}
& \gtrsim \prod_{\substack{I \subset\{2, \ldots, n\}, \# I=m-1}} \prod_{j \in I}\left(\left(\alpha_{1}\left(x_{0}\right) \prod_{i \in I} \alpha_{i}\left(x_{0}\right)\right)^{-\frac{1}{q}} \alpha_{j}\left(x_{0}\right)^{-\frac{n}{p}+\frac{m}{q}}\right) \\
& =\alpha_{1}\left(x_{0}\right)^{-\frac{m-1}{q}\left(\begin{array}{c}
n-1 \\
m-1
\end{array}\right)}\left(\alpha_{2}\left(x_{0}\right) \cdots \alpha_{n}\left(x_{0}\right)\right)^{\left(-\frac{n}{p}+\frac{1}{q}\right)\left(\begin{array}{c}
n-2 \\
m-2
\end{array}\right)} \\
& \sim \alpha_{1}\left(x_{0}\right)^{-\frac{m-1}{q}\left(\begin{array}{c}
n-1 \\
m-1
\end{array}\right)+\left(\left(\frac{n}{p}-\frac{1}{q}\right)\left(\begin{array}{c}
n-2 \\
m-2
\end{array}\right)\right.}=\alpha_{1}\left(x_{0}\right)^{\left(\frac{n}{p}-\frac{n}{q}\right)\left(\begin{array}{c}
n-2 \\
m-2
\end{array}\right)}
\end{aligned}
$$

and then

$$
\alpha_{1}\left(x_{0}\right) \gtrsim 1 .
$$




\subsection{Proof of (2.2) in the case $n q \leq p$}

Let $n q \leq p$. We start with an example of a Morrey function.

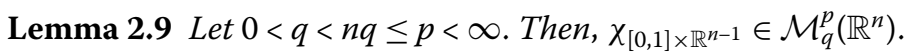

Proof We calculate

$$
\left\|\chi_{[0,1] \times \mathbb{R}^{n-1}}\right\|_{\mathcal{M}_{q}^{p}}=\sup _{R>0} R^{\frac{n}{p}-\frac{n}{q}} \min (1, R)^{\frac{1}{q}} R^{\frac{n-1}{q}}=\sup _{R>0} R^{\frac{n}{p}-\frac{1}{q}} \min (1, R)^{\frac{1}{q}}=1<\infty .
$$

Using Lemma 2.9, we obtain the following estimate on the lower bound of the singular values of matrices.

Lemma 2.10 Let $0<a_{1} \leq \cdots \leq a_{n}$. We assume that $D=\operatorname{diag}\left(a_{1}, a_{2}, \ldots, a_{n}\right)$ induces $a$ bounded composition operator on the Morrey space $\mathcal{M}_{q}^{p}\left(\mathbb{R}^{n}\right)$ with the operator norm $M$. Moreover, if $0<q<n q \leq p<\infty$, then, $a_{1} \geq M^{-\frac{p}{n}}$.

Proof Note that $\chi_{[0,1] \times \mathbb{R}^{n-1}} \circ\left(a_{1} E\right)=\chi_{\left[0, a_{1}-1\right.}{ } \mathbb{R}^{n-1}=\chi_{[0,1] \times \mathbb{R}^{n-1}} \circ D$. Using scaling, we calculate

$$
\begin{aligned}
a_{1}{ }^{-\frac{n}{p}}\left\|\chi_{[0,1] \times \mathbb{R}^{n-1}}\right\|_{\mathcal{M}_{q}^{p}} & =\left\|\chi_{[0,1] \times \mathbb{R}^{n-1}} \circ\left(a_{1} E\right)\right\|_{\mathcal{M}_{q}^{p}} \\
& =\left\|\chi_{[0,1] \times \mathbb{R}^{n-1}} \circ D\right\|_{\mathcal{M}_{q}^{p}} \\
& \leq M\left\|\chi_{[0,1] \times \mathbb{R}^{n-1}}\right\|_{\mathcal{M}_{q}^{p}}
\end{aligned}
$$

Thus, according to Lemma 2.9, this is the desired result.

Using Lemmas 2.3 and 2.10, we conclude that (2.2) holds.

\section{Examples}

In this section, we present some examples and counterexamples. In Example 3.1, the mapping inducing the composition operator satisfies the assumption in Theorem 1.5. In Example 3.2, the mapping inducing the composition operator does not satisfy the assumption in Theorem 1.5; however, the composition operator is bounded on Morrey spaces. Example 3.3 presents a counterexample of cases $n \geq 2$ and $q=p$ in Theorem 1.6.

Example 3.1 The affine map $\varphi$, written as $\varphi(x)=A x+b$ for some $A \in \mathrm{GL}(n ; \mathbb{R})$ and $b \in \mathbb{R}^{n}$ induces the composition operator $C_{\varphi}$ bounded on the Morrey space $\mathcal{M}_{q}^{p}\left(\mathbb{R}^{n}\right)$ whenever $0<q \leq p<\infty$. This follows from the fact that mapping $\varphi$ satisfies the assumption of Theorem 1.5 .

Example 3.2 Let $n=1$ and $1<p<\infty$. Then, the composition operator induced by $\varphi: \mathbb{R} \rightarrow$ $\mathbb{R}$,

$$
\varphi(x) \equiv \begin{cases}e^{x}-1, & \text { if } x \geq 0 \\ x, & \text { if } x<0\end{cases}
$$

is bounded on $\mathcal{M}_{1}^{p}(\mathbb{R})$ and $\varphi$ satisfies the volume estimate (1.2); however, $\varphi$ is not Lipschitz. 
Here, we prove that the composition mapping $C_{\varphi}$ induced by $\varphi: \mathbb{R} \rightarrow \mathbb{R}$ is bounded on $\mathcal{M}_{1}^{p}(\mathbb{R})$. We will prove the inequality

$$
(b-a)^{\frac{1}{p}-1} \int_{a}^{b}\left|C_{\varphi} f(x)\right| \mathrm{d} x \lesssim\|f\|_{\mathcal{M}_{1}^{p}}
$$

for any $-\infty<a<b<\infty$. Here, when $a<0 \leq b$, we calculate

$$
\begin{aligned}
(b-a)^{\frac{1}{p}-1} \int_{a}^{b}|f(x)| \mathrm{d} x & \leq(-a)^{\frac{1}{p}-1} \int_{a}^{0}\left|C_{\varphi} f(x)\right| \mathrm{d} x+b^{\frac{1}{p}-1} \int_{0}^{b}\left|C_{\varphi} f(x)\right| \mathrm{d} x \\
& \leq\|f\|_{\mathcal{M}_{1}^{p}}+b^{\frac{1}{p}-1} \int_{0}^{b}\left|C_{\varphi} f(x)\right| \mathrm{d} x .
\end{aligned}
$$

Then, we may assume that $0 \leq a<b$.

Now, we check inequality (3.1). If $0<b-a \leq 1$, through the change of variable $y=e^{x}-1$ and due to the fact that $e^{b}-e^{a} \sim e^{a}(b-a)$, we obtain

$$
\begin{aligned}
(b-a)^{\frac{1}{p}-1} \int_{a}^{b}\left|C_{\varphi} f(x)\right| \mathrm{d} x & =(b-a)^{\frac{1}{p}-1} \int_{e^{a}-1}^{e^{b}-1}|f(y)| \frac{\mathrm{d} y}{y+1} \\
& \leq\left\{e^{a}(b-a)\right\}^{\frac{1}{p}-1} \cdot e^{-\frac{a}{p}} \int_{e^{a}-1}^{e^{b}-1}|f(y)| \mathrm{d} y \lesssim\|f\|_{\mathcal{M}_{1}^{p}}
\end{aligned}
$$

Furthermore, when $b-a>1$, or equivalently, $(b-a)^{-1}<1$, we calculate

$$
\begin{aligned}
(b-a)^{\frac{1}{p}-1} \int_{a}^{b}\left|C_{\varphi} f(x)\right| \mathrm{d} x & \leq \sum_{j=0}^{\infty} \int_{2^{j}-1}^{2^{j+1}-1}|f(y)| \frac{\mathrm{d} y}{y+1} \\
& \leq \sum_{j=0}^{\infty} 2^{-\frac{j}{p}} \cdot\left(2^{j}\right)^{\frac{1}{p}-1} \int_{2^{j}-1}^{j^{j+1}-1}|f(y)| \mathrm{d} y \lesssim\|f\|_{\mathcal{M}_{1}^{p}}
\end{aligned}
$$

as desired.

Example 3.3 Let

$$
\varphi\left(x_{1}, x_{2}\right) \equiv\left(x_{1}^{3}+x_{1}, \frac{x_{2}}{3 x_{1}^{2}+1}\right)
$$

be a diffeomorphism on $\mathbb{R}^{2}$. Let us consider the boundedness of $C_{\varphi}$ on $\mathcal{M}_{q}^{p}\left(\mathbb{R}^{2}\right)$. In the case of $p=q, C_{\varphi}$ is bounded, $D \varphi\left(x_{1}, x_{2}\right)$ has determinant 1 . In contrast, in the case of $p>q$, $C_{\varphi}$ is not bounded; in fact, the first component is not Lipschitz.

\section{Boundedness of composition operators on weak type spaces}

To prove Theorem 1.13, we use the following identity.

Remark 4.1 Through a simple calculation, we have $\left\|\chi_{E}\right\|_{\mathrm{W} B}=\left\|\chi_{E}\right\|_{B}$ for all measurable sets $E$ in $\mathbb{R}^{n}$. 
Proof of Theorem 1.13 First, we assume that the composition operator $C_{\varphi}$ is bounded on $\mathrm{W} B\left(\mathbb{R}^{n}\right)$, that is, there exists a constant $K$ such that the estimate

$$
\left\|C_{\varphi} f\right\|_{\mathrm{W} B} \leq K\|f\|_{\mathrm{W} B}
$$

holds for any $f \in \mathrm{W} B\left(\mathbb{R}^{n}\right)$. Then, upon choosing $f=\chi_{E}$, the estimate

$$
\left\|\chi_{\varphi^{-1}(E)}\right\|_{\mathrm{W} B}=\left\|C_{\varphi} \chi_{E}\right\|_{\mathrm{W} B} \leq K\left\|\chi_{E}\right\|_{\mathrm{W} B}
$$

holds. By using Remark 4.1, we conclude that

$$
\left\|\chi_{\varphi^{-1}(E)}\right\|_{B} \leq K\left\|\chi_{E}\right\|_{B}
$$

Second, we assume condition (1.4). Considering $E=\left\{x \in \mathbb{R}^{n}:|f(x)|>\lambda\right\}$ for a function $f \in \mathrm{WB}\left(\mathbb{R}^{n}\right)$, we have

$$
\left\|C_{\varphi} f\right\|_{\mathrm{W}_{B}}=\sup _{\lambda>0} \lambda\left\|\chi_{\varphi^{-1}(\{x \in X: f(x) \mid>\lambda\})}\right\|_{B} \leq K \sup _{\lambda>0} \lambda\left\|\chi_{\{x \in X:|f(x)|>\lambda\}}\right\|_{B}=\|f\|_{\mathrm{W}_{B}} .
$$

Finally, the equation

$$
\left\|C_{\varphi}\right\|_{\mathrm{W} B \rightarrow \mathrm{W} B} \leq \sup _{E} \frac{\left\|\chi_{\varphi^{-1}(E)}\right\|_{B}}{\left\|\chi_{E}\right\|_{B}}
$$

is trivial. According to the definition of the operator norm $\|\cdot\| \mathrm{W}_{B \rightarrow \mathrm{W} B}$,

$$
\left\|C_{\varphi}\right\|_{\mathrm{W} B \rightarrow \mathrm{W} B} \geq \sup _{E} \frac{\left\|\chi_{\varphi^{-1}(E)}\right\|_{B}}{\left\|\chi_{E}\right\|_{B}} .
$$

Combining estimates (4.1) and (4.2), we obtain equation (1.5).

The weak type spaces generated by the Banach lattice are essential.

Definition 4.2 We say that a Banach space $\left(B\left(\mathbb{R}^{n}\right),\|\cdot\|_{B}\right)$ contained in $L^{0}\left(\mathbb{R}^{n}\right)$ is a Banach lattice if the inequality $\|f\|_{B} \leq\|g\|_{B}$ holds for all $f, g \in B\left(\mathbb{R}^{n}\right)$ that satisfy $|f| \leq|g|$ almost everywhere.

Remark 4.3 If $B\left(\mathbb{R}^{n}\right)$ is a Banach lattice (see Definition 4.2), then $B\left(\mathbb{R}^{n}\right)$ is embedded in $\mathrm{W} B\left(\mathbb{R}^{n}\right)$.

Now, as the special case of the Morrey space $B\left(\mathbb{R}^{n}\right)=\mathcal{M}_{q}^{p}\left(\mathbb{R}^{n}\right)$, in Theorem 1.13, we have Theorem 1.10.

In Theorem 1.10, through real interpolation, it is known that

$$
\mathrm{WM}_{q}^{p}\left(\mathbb{R}^{n}\right)=\left[\mathcal{M}_{q r}^{p r}\left(\mathbb{R}^{n}\right), L^{\infty}\left(\mathbb{R}^{n}\right)\right]_{1-r, \infty}
$$

as long as $1<q \leq p<\infty$ and $0<r<1$ satisfy $q r>1$ (see [4]). Here, as the $L^{\infty}\left(\mathbb{R}^{n}\right)$ boundedness of the composition operators is trivial and the $\mathcal{M}_{q}^{p}\left(\mathbb{R}^{n}\right)$-boundedness and $\mathcal{M}_{q r}^{p r}\left(\mathbb{R}^{n}\right)$-boundedness of composition operators, for $r>0$, are equivalent owing to the fact that $\left|C_{\varphi} f\right|^{r}=C_{\varphi}\left[|f|^{r}\right]$ for mapping $\varphi$, we obtain that the boundedness " ${ }^{~} C_{\varphi}: \mathcal{M}_{q}^{p}\left(\mathbb{R}^{n}\right) \rightarrow$ $\mathcal{M}_{q}^{p}\left(\mathbb{R}^{n}\right)$ implies the boundedness $C_{\varphi}: \mathrm{W} \mathcal{M}_{q}^{p}\left(\mathbb{R}^{n}\right) \rightarrow \mathrm{W} \mathcal{M}_{q}^{p}\left(\mathbb{R}^{n}\right)^{\prime \prime}$. 


\section{Acknowledgements}

The authors are thankful to Professor Ken-ichi Bannai at Keio University for giving us a chance to consider the problem.

\section{Funding}

This work was supported by a JST CREST Grant (Number JPMJCR1913, Japan). This work was also supported by the RIKEN Junior Research Associate Program. The second author is supported by a Grant-in-Aid for Young Scientists Research (No. 19K14581), Japan Society for the Promotion of Science. The fourth author is supported by a Grant-in-Aid for Scientific Research (C) (19K03546), Japan Society for the Promotion of Science.

Availability of data and materials

No data and material were used to support this study.

\section{Competing interests}

The authors declare that they have no competing interests.

\section{Authors' contributions}

The four authors contributed equally to this paper. All of them read the whole manuscript and approved the content of the paper.

\section{Author details}

'Department of Mathematics, Faculty of Science and Technology, Keio University, 3-14-1 Hiyoshi, Kohoku-ku, Yokohama 223-8522, Japan. ${ }^{2}$ Center for Advanced Intelligence Project, RIKEN, Tokyo, Japan. ${ }^{3}$ Department of Mathematics, Chuo University, 1-13-27, Kasuga, Bunkyo-ku, Tokyo 112-8551, Japan. ${ }^{4}$ Department of Engineering for Production and Environment, Graduate School of Science and Engineering, Ehime University, 3 Bunkyo-cho, Matsuyama, Ehime 790-8577, Japan.

\section{Publisher's Note}

Springer Nature remains neutral with regard to jurisdictional claims in published maps and institutional affiliations.

\section{Received: 2 February 2021 Accepted: 26 March 2021 Published online: 09 April 2021}

\section{References}

1. Arora, S.C., Datt, G., Verma, S.: Composition operators on Lorentz spaces. Bull. Aust. Math. Soc. 76(2), 205-214 (2007)

2. Castillo, R.E., Vallejo Narvaez, F.A., Ramos Fernandez, J.C.: Multiplication and composition operators on weak $L_{p}$ spaces. Bull. Malays. Math. Sci. Soc. 38(3), 927-973 (2015)

3. Cui, Y., Hudzik, H., Kumar, R., Maligranda, L.: Composition operators in Orlicz spaces. J. Aust. Math. Soc. 76(2), 189-206 (2004) (English summary)

4. Cwikel, M., Gulisashvili, A.: Interpolation on families of characteristic functions. Stud. Math. 138(3), 209-224 (2000)

5. Evseev, N., Menovschikov, A.: Bounded operators on mixed norm Lebesgue spaces. Complex Anal. Oper. Theory 13(5), 2239-2258 (2019)

6. Evseev, N.A.: A bounded composition operator on Lorentz spaces. Mat. Zametki 102(6), 836-843 (2017) (Russian), translation in Math. Notes 102(5-6), 763-769 (2017)

7. Evseev, N.A., Menovshchikov, A.V.: The composition operator on mixed-norm Lebesgue spaces. Mat. Zametki 105(6), 816-823 (2019) (Russian), translation in Math. Notes 105(5-6), 812-817 (2019)

8. Grafakos, L.: Classical Fourier Analysis, 3rd edn. Texts in Mathematics, vol. 249. Springer, New York (2014)

9. Ikeda, M., Ishikawa, I., Sawano, Y.: Composition operators on reproducing kernel Hilbert spaces with analytic positive definite functions. arXiv:1911.11992

10. Ishikawa, I., Fujii, K., Ikeda, M., Hashimoto, Y., Kawahara, Y.: Metric on nonlinear dynamical systems with Perron-Frobenius operators. Adv. Neural Inf. Process. Syst. 31, 911-919 (2018)

11. Kawahara, Y.: Dynamic Mode Decomposition with Reproducing Kernels for Koopman Spectral Analysis. Neural Information Processing Systems, Advances in Neural Information Processing Systems, vol. 29 (2016)

12. Koopman, B.O.: Hamiltonian systems and transformation in Hilbert space. Proc. Natl. Acad. Sci. 17(5), 315-318 (1931)

13. Kumar, R.: Composition operators on Orlicz spaces. Integral Equ. Oper. Theory 29(1), 17-22 (1997)

14. Raj, K., Sharma, S.K.: Composition operators on Musielak-Orlicz spaces of Bochner type. Math. Bohem. 137(4), 449-457 (2012)

15. Sawano, Y.: A non-dense subspace in $\mathcal{M}_{q}^{p}$ with $1<q<p<\infty$. Trans. A. Razmadze Math. Inst. 171(3), $379-380$ (2017)

16. Singh, R.K.: Composition operators induced by rational functions. Proc. Am. Math. Soc. 59(2), 329-333 (1976) 